\title{
Cognitive chrono-ethnography lite
}

\author{
Masato Nakajima, ${ }^{\mathrm{a},}$, Kosuke C Yamada a and Muneo Kitajima ${ }^{\mathrm{a}}$ \\ ${ }^{a}$ Center for Service Research, National Institute of Advanced Industrial Science and Technology (AIST) \\ 2-3-26 Aomi, Kouto-Ku, Tokyo, Japan
}

\begin{abstract}
Conducting field research facilitates understanding human daily activities. Cognitive Chrono-Ethnography (CCE) is a study methodology used to understand how people select actions in daily life by conducting ethnographical field research. CCE consists of measuring monitors' daily activities in a specified field and in-depth interviews using the recorded videos afterward. However, privacy issues may arise when conducting standard CCE with video recordings in a daily field. To resolve these issues, we developed a new study methodology, CCE Lite. To replace video recordings, we created pseudo-first-personview (PFPV) movies using a computer-graphic technique. The PFPV movies were used to remind the monitors of their activities. These movies replicated monitors' activities (e.g., locomotion and change in physical direction), with no human images and voices. We applied CCE Lite in a case study that involved female employees of hotels at a spa resort. In-depth interviews while showing the PFPV movies determined service schema of the employees (i.e., hospitality). Results indicated that using PFPV movies helped the employees to remember and reconstruct the situation of recorded activities.
\end{abstract}

Keywords: Cognitive Chrono-Ethnography, privacy issues, behavior measurement, in-depth interview, pseudo-first-personview movies

*Corresponding author. E-mail: masato-nakajima@aist.go.jp 


\section{Introduction}

We are interested in understanding how human beings acquire and develop knowledge and skills through their repeated daily activities. Cognitive Chrono-Ethnography (CCE) was developed as a study methodology to understand how human beings select actions unconsciously or consciously in daily life and they have chronologically modified their behavior to the present style by conducting an ethnographical field research [3,5]. This methodology is designed by taking into account human beings' cognitive-behavioral processing under real-time constraints. It utilizes the nature of memory encoding when an action is taken and memory recall when an interview is conducted afterward to reveal the interviewee's memory at the time the actions were selected (i.e., encoding specificity principle of memory) [2].

In order to fully utilize these characteristics of memory, the CCE studies that have been conducted thus far video-recorded the monitors' behavior (e.g., when they watched baseball games at the stadium [1], when they were driving a car [4], or when they walked through train stations [6]), and used the recorded video in the after-event interview sessions as retrieval cues to help the monitors reconstruct their memory structure that was active when they were video-recorded. Results indicated that this procedure (i.e., behavior video-recording, and memory reconstruction using the recorded video) is very effective for understanding peoples' action selection processes.

A CCE study consists of two stages: behavioral measurement by conducting an ethnographical field research, and a series of in-depth interviews that uses the records in the behavioral measurement. However, practical issues may arise during a standard CCE study when it utilizes video recordings. Privacy issues may arise when it is difficult to obtain informed consent from the people who are to be video-recorded. This could impose some restrictions on the detailed procedures of conducting behavioral measurement and in-depth interviews.

\subsection{Need to develop privacy-issue-free CCE}

The purpose of this paper is to present a case in which adaptation of CCE was required due to the privacy issues and to demonstrate how we solved it. The study field was "provision of service by employees at a hot spring resort in Japan." The purpose of the study was to understand the knowledge in- volved in situated service communication. We conducted a preliminary survey and found that superior employees have knowledge that qualitatively differs from that of ordinary employees. Ordinary employees are expected to acquire this knowledge. Their employers are anxious to know what this knowledge is, and an efficient and effective procedure to help ordinary employees acquire it. However, superior employees acquire such knowledge through their work career. We needed to extract the knowledge that superior employees have. Thus, the problem was knowledge extraction from superior employees. However, it was difficult to extract the knowledge of the superior employees by a standard way to ask questions because they have already become tacit.

To solve the problem, CCE should be utilized. We could have conducted this study in the same way as the previous CCE studies, by having the employees wear ear-mounted cameras to record their activities. An ear-mounted camera attached to an employee will record his/her situated service communication from this employee's first-person-view. Showing the videos will help the employee recall and reconstruct concisely his/her activities in in-depth interviews.

However, we had to give up the idea of using the standard CCE procedure because there were severe privacy issues in the field in question. It was likely that the video recordings would include people's images and voices with whom the employee was interacting. These images and voices should neither be recorded nor be used as data for the study unless their use was accepted by the recorded persons. Recently, ergonomics ethics has required researchers should address privacy issues arising in the laboratory.

In addition to privacy issues, a few problems would arise in behavior measurements. Firstly, direct observation and video recording often make monitors and observers feel some kinds of mental stress. Recorded monitors are often unable to concentrate on their daily activities by just being observed. Some guests with whom the monitors are interacting would feel unease and discomfort because they feel they are recorded when they notice the ear-mounted camera. This would result in unnatural service communication. Secondly, observers often find it difficult to record monitors' activities that involve a wide range of movement. The adapted CCE proposed in this paper would help alleviate these problems as well as the privacy issues. 


\section{CCE Lite : Privacy-issue-free version of CCE}

In this paper, we present CCE Lite to resolve privacy issues that may arise in a situation in which nonmonitors' images and voices may be recorded. An innovative technique was introduced to the design of privacy-issue-free version of CCE, CCE Lite, which uses pseudo-first-person-view (PFPV) movies for retrieval cues in place of the video-recorded data. We adopted an "indoor pedestrian tracking system (PDR)" to record monitors' locomotion during the behavior measurement.

\subsection{Using a pseudo-first-person-view movie}

A key procedure of the CCE methodology is to conduct in-depth interviews using recorded video movies, which would determine the knowledge of hospitality that employees have. However, we could not use video recording of employees' activities. Therefore, in this study, to replace these movies (i.e., real FPV movies), we created pseudo-first-personview (PFPV) movies using a computer graphic technique [7]. PFPV movies replicate monitors' activities (e.g., movement and change in physical direction) from one's first-person-view (see Figure 1). These movies did not include people's images and voices. PFPV movies were used in in-depth interviews as contextual cues to stimulate employees' memory of their activities. It was expected that information about movement would help the employees recall and reconstruct concisely what they did and where they did it.

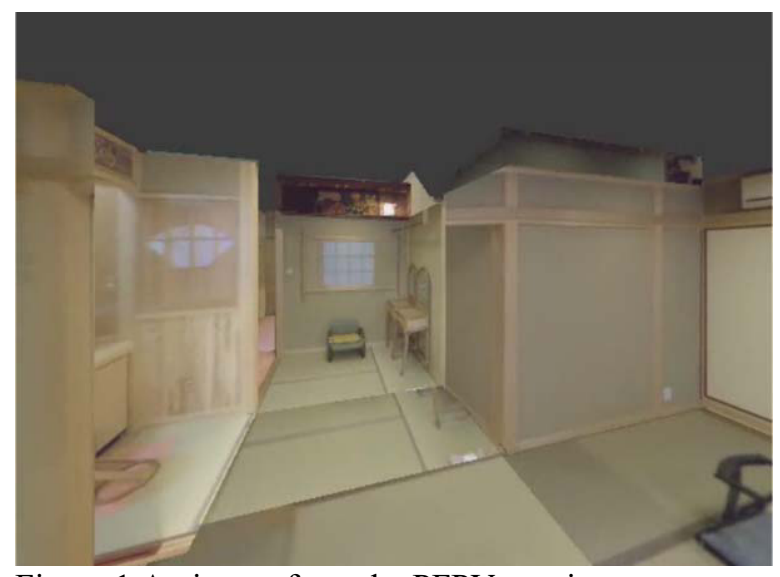

Figure 1 An image from the PFPV movies

\subsection{Creating the PFPV movies}

We adopted the following two innovative techniques, PDR and in-situ 3D modeling, to create the PFPV movies.

\subsubsection{Indoor pedestrian tracking system}

An indoor pedestrian tracking system was adopted for recording employees' activities in the field research $[8,9]$. Pedestrian dead-reckoning (PDR) is a technique to calculate the location of a moving object in the $3 \mathrm{D}$ coordinates by having it mount a small wearable sensor that can quantitatively record time as well as the subject's location, physical direction, and relative locomotion.

PDR has several merits. The PDR sensor is light and small enough not to distract employees from their activities (see Figure 2). It records the device's location in the $3 \mathrm{D}$ coordinates and the time. Therefore, there is no need for observers to follow the employees during the observation and to review video records to extract these data.

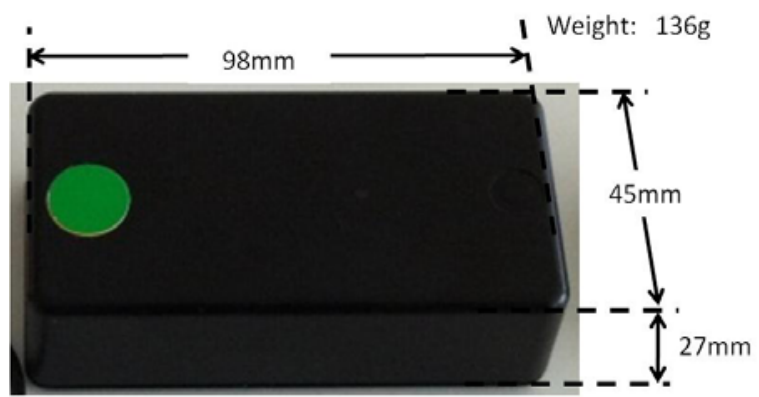

Figure 2 PDR sensor

\subsubsection{In-situ $3 D$ indoor model}

In-situ 3D indoor modeling was used to create a photorealistic indoor 3D model of a building from 2D pictures by applying computer graphics techniques [10]. Figure 3 illustrates a bird's-eye view of the global indoor 3D model of a hotel. The model was used as a 3D map to understand when and where the employees were by integrating quantitative data recorded by PDR with the 3D model. Figure 4 illustrates one of the local indoor 3D models of the room (e.g., a pantry) of the hotel. This model was used for producing pseudo-first-person-view movies. Interiors (e.g., the wall, furniture) in the room were restored in $3 \mathrm{D}$. 


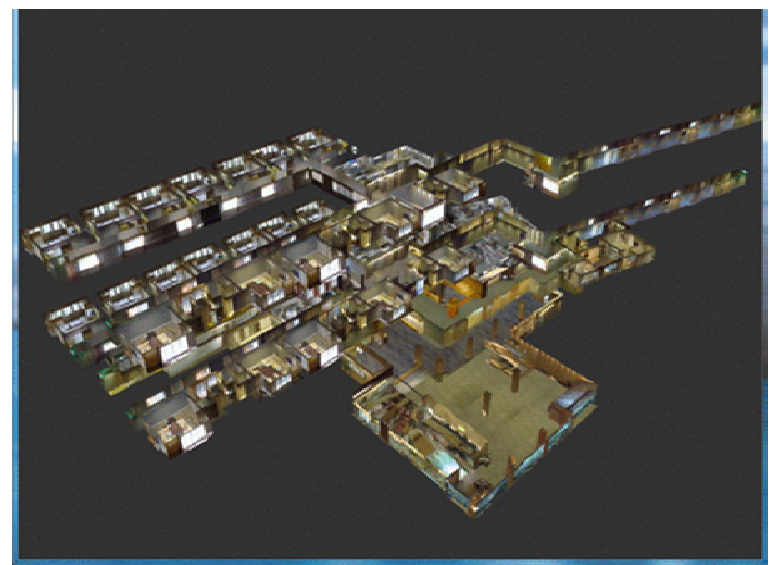

Figure 3 A bird's-eye view of the global indoor 3D model of a hotel.

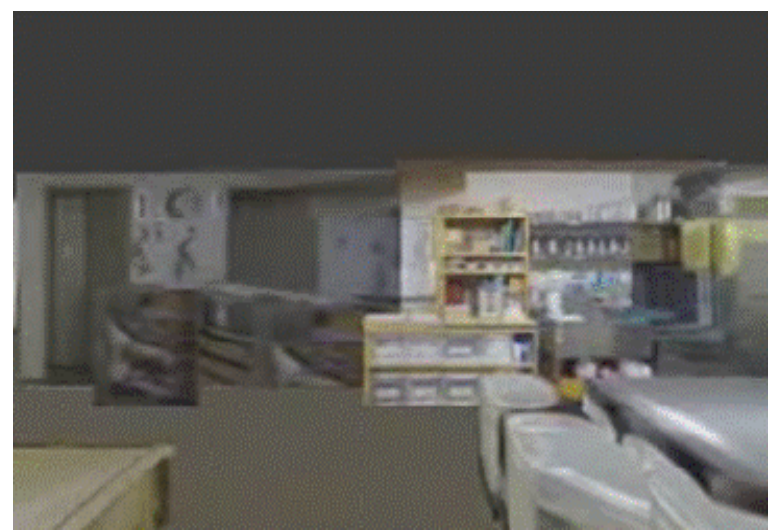

Figure 4 A first-person-view of the local indoor 3D model of a pantry.

\section{Case study of CCE Lite: To verify the effect of using PFPV movies}

We used the CCE Lite with hotel employees at a spa resort as a representative Japanese hospitality industry to understand tacit knowledge of hospitality. In this case study, we aimed to verify how accurately and completely the employees were able to recall the situations and their activities when we used PFPV movies. The PFPV movies resolved the privacy issues. However, we were concerned that employees might not remember and reconstruct the situation because the PFPV movies lacked some dimensions of the information. For example, the movies do not include actual interactions with the guests, guests' features, the few pieces of furniture in the room, and changes in brightness accompanying temporal progress. We expected to extract appropriately the employees' tacit knowledge and determine how they acquired that knowledge during the in-depth interviews while showing the PFPV movies. Therefore, it was necessary to determine how much and how correctly the employees could recall the situation by using the PFPV movies.

\subsection{Behavior measurement}

This study's monitors were eight female room maids who had different careers at three Japanese hotels at Kinosaki Onsen spa resort in Hyogo Prefecture, Japan. Selection of the employees was not controlled systematically. Their career ranged from two to more than thirty years.

Employees engaged in their daily work as usual for one or two days. Each employee wore a PDR sensor on her back (see Figure 5). The PDR sensor was fixed in a girdle, not to be seen from the guests. Therefore, the observation could be conducted in a natural situation.

Each day they were asked to write a diary memo about their work after they finished working of the day. Their memos were used in the in-depth interviews to help them recall their activities.

We created PFPV movies of each employee by integrating the data recorded by PDR with the 3D model of each hotel.

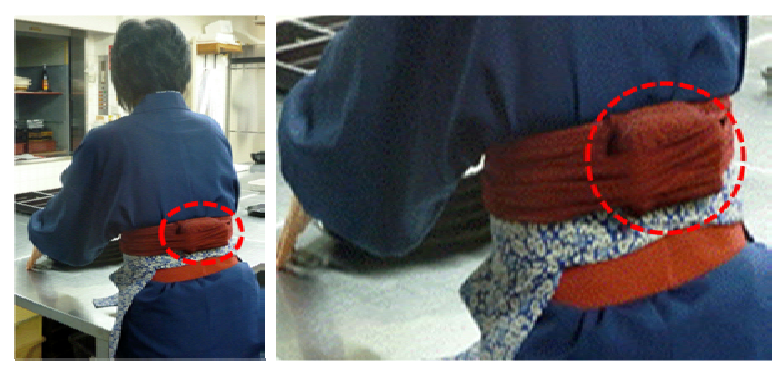

Figure 5 The study monitor wearing a PDR sensor on her back. Circle broken lines indicate the location of the PDR sensor.

\subsection{In-depth interviews}

In-depth interviews were conducted three times with each individual employee. Each interview took 60 minutes. The first interview was concerned about the general flow of daily work without using PFPV movies. In the second interview, we asked about the service characteristics for guests, events, and situations while showing the PFPV movies to each employee. In the third interview, we asked employees 
how they acquired knowledge about service, while showing the PFPV movies.

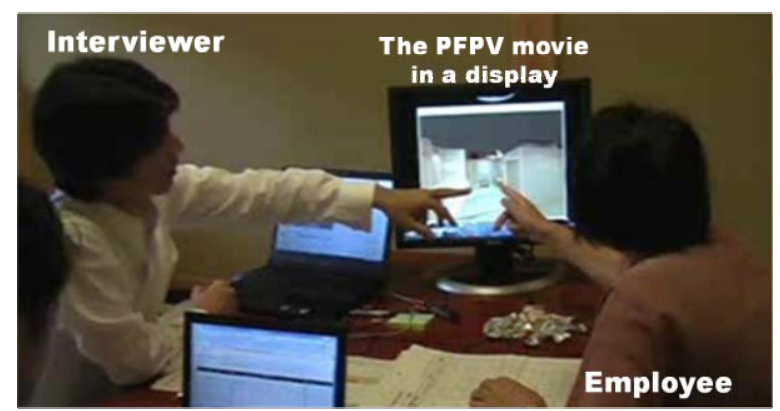

Figure 6 A scene from an in-depth interview session with PFPV movies shown in the display. The close-up snap-shot of this PFPV movie in this figure is shown in Figure 1.

\section{Results}

Results indicated that the PFPV movies helped the employees remember and reconstruct their activities in the field observations and many related matters (e.g., service schemata and skills). Almost all the employees indicated that the PFPV movies were effective for them to remind them of the guests' characteristics and information (e.g., age, gender, and personality) when they provided their services. They then began to tell how they used the schemata for the guests, depending on the situation in which they provided their services. In addition, we found that those with longer careers had more various types of service skills and schemata that they used for guests.

\subsection{Schemata of situated service communications for hotel employees}

We extracted three kinds of schemata that were required for the hotel employees: for a person, for a situation, and for the service process. Schemata for a person relate to individual information about the guests (e.g., age, gender, and personality), their traveling styles (e.g., number of guests, family, and couples), and the purposes of their travel (e.g., sightseeing, shopping, and hot spring). Schemata for the situation relate to such information as time and place. Schemata for the service process relate to such services as guiding guests to their rooms and setting the table for dinner.
We identified general schemata that every employee had and peculiar schemata that part of the employees developed depending on their work environments and careers. Some employees provided efficient service in their own way, using their knowledge. Results indicated that the variety and quality of services increased with the length of the career.

\subsection{Process of acquiring service schemata for hotel employees}

We verified that the acquisition of service schemata for employees involved the process of human development from unspecialized to specialized. Shortcareer employees would have acquired the patterned minimum schemata for service provision with the advice of superior employees and trainers. Employees' knowledge and skill become subdivided as their careers become longer. As a result, they can provide services that are sure to satisfy their guests.

We found that some employees originally strived to improve and devised their services based on their mistakes made in actual service situations. In this case, the reactions of fellow workers, superior employees, and supervisors were helpful. Most efforts to acquire the knowledge and skills to provide services were triggered when they made mistakes and were cautioned by their guests and supervisors.

However, it is difficult to understand the details of triggers to acquire knowledge because they learned them unconsciously. From the methodological point of view, it is necessary to devise a way to ask questions and construct interviews to obtain useful information.

\section{Conclusion}

In this study, we developed CCE Lite, a modified version of $\mathrm{CCE}$, to resolve privacy issues that may arise when guests' images and voices may be recorded. We verified that the CCE Lite could reliably extract many pieces of employees' tacit information. PFPV movies helped them remember and reconstruct the situation and related matters at the time their activities were recorded.

As to the ergonomics ethics, the CCE Lite would expand the adaptable fields to be observed, which has been restricted by the ergonomics ethics. In addition, it would help alleviate mental and physical stresses of monitors and observers. Therefore, in particular, this approach could be adaptable for the fields where pri- 
vacy issues could arise and employees frequently move indoors.

In future studies, we should investigate how exhaustively the CCE Lite extracts tacit information of superior employees under systematically controlled study conditions. The information will be utilized for training new comers and ordinary employees to provide high quality services.

\section{Acknowledgement}

This study was supported by the Ministry of Economy, Trade and Industry.

\section{References}

[1] E. Someya, M. Kitajima, H. Tahira and T. Kajikawa. Project $\mathrm{B} * \mathrm{~B}$ : Develop-mental processes of fan loyalty for the professional baseball team 'The Hokkaido Nippon-Ham Fighters. Proceedings of the 2009 North American Society for Sport Management Conference, 2009, pp. 364-365.

[2] G. Godden and A. Baddeley, Con-text-dependent memory in two natural environments: On land and underwater. British Journal of Psychology, 6, 1975, pp. 355-369.

[3] M. Kitajima, H. Tahira, S. Takahashi, and T. Midorikawa, Understanding Tourist's in situ Behavior: a Cognitive ChronoEthnography Study of Visitors to a Hot Spring Resort, Journal of Quality Assurance in Hospitality and Tourism, in press.

[4] M. Kitajima, M. Akamatsu, Y. Maruyama, K. Kuroda, K Katou, S. Kitazaki, Y. Minowa, K. Inagaki, and T. Kajikawa, Information for Helping Drivers Achieve Safe and Enjoyable Driving: An On-Road Observational Study. Proceedings of the Human Factors and Ergonomics Society 53rd Annual Meeting, 2009, pp. 1801-1805.

[5] M. Kitajima, M. Nakajima and M. Toyota, Cognitive ChronoEthnography: A Method for Studying Behavioral Selections in Daily Activities, Proc. of Annual Meeting of Human Factors and Ergonomics Society, 2010, pp.1732-1736

[6] M. Kitajima, T. Kumada, M. Akamatsu, H. Ogi and H. Yamazaki, Effect of Cognitive Ability Deficits on Elderly Passengers' Mobility at Railway Stations - Focusing on Attention, Working Memory, and Planning. The 5th International Conference of the International Society for Gerontechnology, 2005.

[7] M. Kourogi, N. Sakata, T. Okuma, and T. Kurata, Indoor/Outdoor Pedestrian Navigation with an Embedded GPS/RFID/Self-Contained Sensor System. Proc. of Int. Conf. on Artificial Reality and Telexistence, 2006, pp. 1310-1321.

[8] M. Kourogi, T. Ishikawa, and T. Kurata, A Method of Pedestrian Dead Reckoning Using Action Recognition. IEEE/ION PLANS 2010 Position Location and Navigation Symp., 2010, pp. 85-89.

[9] T. Ishikawa, M. Kourogi, T. Okuma, and T. Kurata, Economic and Synergistic Pedestrian Tracking System for Indoor Environments. In Proc. of Int. Conf. on Soft Computing and Pattern Recognition, 2009, pp. 522 - 527.

[10] T. Ishikawa, T. Kalaivani, M. Kourogi, A. P. Gee, W. Mayol, K. Jung and T. Kurata, In-Situ 3D Indoor Modeler with a Camera and Self-Contained Sensors. In Proc. 13th Int. Conf. on Human-Computer Interaction, 2009, pp. 454 - 464. 